\title{
No pain, much gain?
}

\author{
Nanette M. Schwann, MD \\ Mark A. Chaney, MD
}

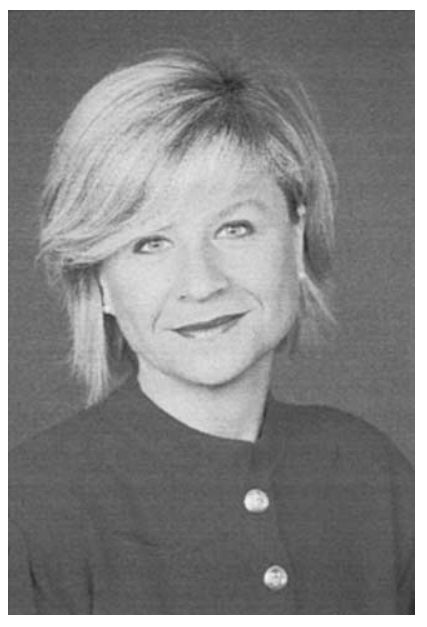

Dr Schwann

See related article on page 1271.

From the Department of Anesthesiology and Cardiothoracic Surgery, ${ }^{a}$ Drexel University College of Medicine, Philadelphia, $\mathrm{Pa}$, and the Department of Anesthesia and Critical Care, ${ }^{\mathrm{b}}$ University of Chicago, Chicago, Ill.

Received for publication June 18, 2003; accepted for publication June 23, 2003.

Address for reprints: Nanette M. Schwann, MD, Drexel University School of Medicine, Mail Stop 310, 245 N 15th St, Philadelphia, PA 19102 (E-mail: schwann@ drexel.edu).

J Thorac Cardiovasc Surg 2003;126:1261-4

Copyright (C) 2003 by The American Association for Thoracic Surgery

$0022-5223 / 2003 \$ 30.00+0$

doi:10.1016/S0022-5223(03)01327-8

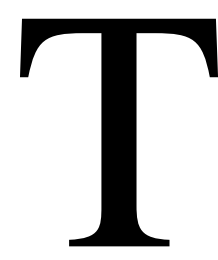

he goal of anesthesia is to erase the reality of the surgical insult and to restore patients as quickly as possible to their premorbid state. The safest and most cost-effective anesthetic technique with the greatest salutary effect on cardiac surgical outcome is a subject of current debate. Economic and social realities have rendered the hemodynamically stable, high-dose, narcotic-based anesthetic technique pioneered by Edward Lowenstein in the early 1970s fiscally prohibitive. In addition, societal expectations for minimally invasive surgery and rapid recovery fuel the growth and ubiquity of fast-track anesthesia for all but the most critical of patients. As a result, shorter-acting anesthetic agents are currently used to restore consciousness and spontaneous unassisted ventilation within hours of termination of the cardiac surgical procedure. With the line between anesthetics for cardiac surgery and anesthetics for other major surgical procedures becoming increasingly faint, the importance of pain control in the early postoperative period is emerging.

The natural history of pain after cardiac surgery remains inadequately described and traditionally dismissed as of minor clinical consequence. Undoubtedly, this perception has evolved from the long history of prolonging general anesthesia into the postoperative period, a practice that is now no longer economically viable. Pain and the ensuing stress response are being increasingly recognized for their contributory role in postoperative complications and their profound cumulative economic effect. ${ }^{1}$ Yet the majority of cardiac intensive care units in the United States still adhere to the antiquated, highly subjective, and inefficient paradigm of intravenous, nurse-administered postoperative analgesia. Few of the regional anesthetic techniques (spinal, epidural, and nerve block) or supplementary adjuvants (nonsteroidal anti-inflammatories and patient-controlled analgesia) widely used in the general surgical population to attenuate postoperative pain have been adopted into mainstream cardiac care. Thus the sophistication, technologic advancements, and customization of postoperative pain management seem to have bypassed one of the largest surgical populations in the world today. This situation requires change.

This month's featured article entitled "Improved pain control after cardiac surgery," coauthored by the surgeon-anesthesiologist team of Robert Dowling, Kenneth Thielmeier, and their associates, emphasizes the need for change and underscores the potential positive consequences that attenuation of pain after cardiac surgery might have on outcome, cost, and patient satisfaction. ${ }^{2}$ This very small (n $=35$ patients) prospective, randomized, blinded, placebo-controlled trial examining intraoperative bilateral intercostal nerve block (and continuous subcutaneous infusion) with either local anesthetic (ropivacaine) or placebo after median sternotomy not surprisingly reveals decreased postoperative consumption of intravenous narcotics and significantly lower visual analog pain scores in patients receiving the local anesthetic. More notably, the authors also demonstrate a shorter hospital length of stay in the treatment group and estimate the potential annual national cost implication at approximately 1.4 billion dollars. However, the attempt to link improved pain control with better clinical outcome was unsuccessful. A likely explanation for the acceptance of the null hypothesis might be attributable to the small sample size, the relatively healthy patient population studied, and the relatively crude respiratory and cardiac end points chosen to investigate. However, this study is important because it focuses our attention on an underappreciated, inade- 
quately studied, and poorly understood aspect of cardiac surgical recovery that, if better understood, might be manipulated to improve morbidity and decrease cost.

\section{The Pain Prism}

Pain is defined as "an unpleasant sensory and emotional experience associated with actual or potential tissue damage." The surgical insult of cardiac surgery can be conceptually divided into 2 main components: that associated with tissue trauma/pain and that associated with the sequelae of cardiopulmonary bypass (CPB). Acute postoperative pain from required surgical exposure is primarily inflammatory in nature, associated with release of chemical mediators, and possibly modified in perception and expression by psychologic and social factors. Classically, the intensity of pain is commensurate with the extent and the location of the soft tissue destruction (with small peripheral incisions eliciting less response in comparison with large central incisions). The degree to which the tissue trauma of sternotomy, with pericardial entry and cardiac manipulation, contributes to the total stress response exhibited by patients undergoing cardiac surgery has not been fully elucidated. It has been known for many years that CPB induces a wide variety of physiologic changes that could be detrimental to patients undergoing cardiac surgery. There is a plethora of validated evidence indicating that the intensity of this stress response during cardiac surgery greatly exceeds that of most other surgical procedures. Studies further suggest that the intensity of this stress response during off-pump coronary revascularization $(\mathrm{OPCAB})$ is less profound when compared with that after similar surgery with the assistance of $\mathrm{CPB}$, yet the presence of significant amounts of inflammatory markers even after OPCAB intimates that $\mathrm{CPB}$ is not the solitary source of postoperative stress. Because of the complexity, interdependency, and overlap of these inflammatory pathways (both pain-related and procedure-related pathways), the teasing out of the precise relative contribution of pain versus procedure to the entire postoperative stress response is difficult.

All types of anesthesia (general or regional) seek to eliminate both components of the surgical injury. General anesthesia remains the predominant technique for patients undergoing cardiac surgery and, by definition, provides analgesia, amnesia-hypnosis, and muscle relaxation, which are necessary for the procedure. Although it is technically feasible to perform coronary surgery through a median sternotomy with regional anesthesia alone, ${ }^{3}$ adequate spontaneous ventilation after mediastinal entry, thoracic entry, or both limits the adoption of this technique to only the healthiest of patients undergoing OPCAB.

Accepting the premise that general anesthesia with mechanical ventilation is necessary for the safe execution of cardiac surgery, then the ideal anesthetic would comprise the following features: complete intraoperative and postoperative elimination of the pain-mediated stress response, attenuation of the stress response to CPB, and quick return of adequate, unassisted, homeostatic bodily functions (eg, respiration, circulation, and cognitive function) at the termination of the surgical procedure. Unfortunately, the cessation of general anesthesia soon after surgical intervention requires the withdrawal of intravenous and inhalation agents, rendering the patient at risk for pain and its detrimental consequences on respiration and hemodynamics.

\section{The Case Against Pain}

Inadequate analgesia, an uninhibited stress response, or both during the postoperative period could lead to many adverse hemodynamic (tachycardia, hypertension, and vasoconstriction), metabolic (increased catabolism), immunologic (impaired immune response), and hemostatic (platelet activation) alterations. In patients undergoing cardiac surgery, perioperative myocardial ischemia is most commonly observed during the immediate postoperative period and is related to outcome. As previously stated, numerous intraoperative factors (including $\mathrm{CPB}$ ) stimulate significant increases in stress response hormones (eg, norepinephrine and epinephrine) that persist into the immediate postoperative period and might contribute to myocardial ischemia observed during this time. Furthermore, postoperative myocardial ischemia could be aggravated by cardiac sympathetic nerve activation, which disrupts the balance between coronary blood flow and myocardial oxygen demand. Aggressive control of postoperative pain with intravenous analgesics in patients after cardiac surgery could potentially decrease morbidity and mortality. ${ }^{4,5}$ In adults undergoing cardiac surgery, intense analgesia (continuous intravenous opioid infusion) during the immediate postoperative period (first 18 hours) decreases the incidence and severity of myocardial ischemia, as diagnosed by means of electrocardiography. ${ }^{4}$ In neonates undergoing cardiac surgery, intense analgesia (continuous intravenous opioid infusion) during the immediate postoperative period (first 24 hours) decreases morbidity and mortality. ${ }^{5}$ Unfortunately, aggressive control of pain with intravenous opioids in this manner does not allow tracheal extubation to occur in the immediate postoperative period.

\section{Regional Anesthetic Techniques}

Although use of specific nerve blocks has been sporadically investigated, most attempts at regional anesthesia supplementation in patients undergoing cardiac surgery have involved spinal intrathecal techniques, epidural techniques, or both. Intrathecal or epidural application of opioids, local anesthetics, or both are attractive alternatives to intravenous opioids in this setting for their potential to produce intense analgesia yet still allow extubation to occur in the immedi- 
ate postoperative period. ${ }^{6}$ In addition to producing intense postoperative analgesia, perioperative use of intrathecal or epidural techniques in patients undergoing cardiac surgery might also beneficially affect outcome through stress response attenuation (not related to analgesia) and induction of thoracic cardiac sympathectomy. ${ }^{6}$

Intrathecal and epidural local anesthetics appear to possess greater efficacy than opioids (administered similarly) in stress response attenuation, perhaps because of their unique mechanism of action, which also contributes to the hypotension associated with their use. Local anesthetics effectively block cardiac sympathetic nerve afferent and efferent fibers, whereas opioids (administered similarly) are unable to do so. In short, application of local anesthetics might benefit patients undergoing cardiac surgery by effectively blocking cardiac sympathetic nerve activity and improving myocardial oxygen supply-demand balance through numerous mechanisms.

Well-controlled clinical investigations attest to the ability of intrathecal opioids to reliably induce intense postoperative analgesia in patients after cardiac surgery. ${ }^{7,8}$ Although use of intrathecal local anesthetics has been investigated, the hemodynamic changes associated with a total spinal make the technique unpalatable to most anesthesiologists. Because of enhanced flexibility, most recent clinical investigations have focused on use of thoracic epidural techniques in patients undergoing cardiac surgery. In contrast to intrathecal techniques (single shot of a drug), the presence of a catheter in the epidural space allows an almost infinite number of possibilities regarding potential drugs (opioids, local anesthetics, or both) and dosing schedules to attain intense postoperative analgesia, stress response attenuation, and/or thoracic cardiac sympathectomy. Administration of epidural local anesthetics (not opioids) can both reliably attenuate the stress response associated with the immediate postoperative period and induce thoracic cardiac sympathectomy. Suggested clinical benefits of either the intrathecal or epidural technique include enhanced postoperative analgesia, improved postoperative pulmonary function, facilitation of early extubation, improved myocardial protection, decreased postoperative arrhythmias, improved postoperative renal function, and decreased postoperative confusion. ${ }^{9-11}$

One must keep in mind, however, that using intrathecal techniques, epidural techniques, or both in patients undergoing cardiac surgery entails risk. The most troubling and undesirable drug effect of intrathecal or epidural local anesthetics is hypotension. Although many have been described, the four clinically relevant undesirable drug effects of intrathecal and epidural opioids are pruritus, nausea and vomiting, urinary retention, and respiratory depression. Excessive respiratory depression might delay tracheal extubation. The most feared complication of intrathecal and epi- dural instrumentation is hematoma formation. Substantial controversy exists regarding the safety of intrathecal instrumentation, epidural instrumentation, or both in patients who will subsequently receive anticoagulation. Substantial controversy also exists regarding whether intrathecal and epidural techniques truly affect morbidity and mortality in patients undergoing cardiac surgery. Essentially, all reports of intrathecal and epidural techniques in patients undergoing cardiac surgery involve small numbers of patients, and few (if any) are well designed. Furthermore, most investigations do not use clinical outcome as a primary end point. Thus there are clear deficiencies in the literature that prohibit definitive analysis of the risk/benefit ratio of intrathecal and epidural techniques in patients undergoing cardiac surgery.

Intercostal nerve block, as described in the current article, has been used extensively and effectively for blunting pain associated with thoracotomy, as well as with chest tube insertion. However, its use as an analgesic adjunct after median sternotomy is less common. The analgesic effect of single-shot intercostal nerve block with ropivacaine is transient, with a duration of action of less than 4 hours. The subcutaneous continuous infusion used in this study, administered through a catheter positioned anterior to the sternal wires, presumably bathes the anterior cutaneous branches of the intercostal nerves at multiple levels, thereby providing a longer-acting analgesic effect. It is important to note that systemic absorption of local anesthetic from an intercostal nerve block is substantial and rapid (when compared with other anatomic sites). Although increased plasma concentrations of local anesthetic are necessary to produce cardiac and neurologic toxicity (ventricular arrhythmia and seizures), such potentially lethal complications should always be considered and total drug dosages adjusted accordingly on the basis of appropriate factors (eg, specific local anesthetic, site of injection, and body weight).

\section{The Future: Multimodal Anesthesia?}

In this era of challenge and great uncertainty for the noble specialty of cardiothoracic surgery, this investigation by Dowling and associates ${ }^{2}$ is notable for its distinctly nonsurgical focus. Although not providing definitive data and proof, their small clinical investigation indicates that what most of us intuitively believe might be true: that the quality of postoperative pain control is likely linked to morbidity (and thus linked to cost). If one were to try to predict the future, the next step to the management of postoperative pain most likely lies in the synthesis of multiple techniques, each inadequate in itself, but when used together exhibiting a significant effect with a much attenuated risk profile. Further study is needed to solidify the link between pain and morbidity and to better quantify the risks of the neuraxial anesthetic techniques in our surgical population. We hope 
that such investigation heralds the beginning of a culture change toward a patient-centered approach to cardiac surgical interventions and investigations (involving surgeons and anesthesiologists) in which the patient's experience is inextricably linked to the definition of quality care and outcome.

\section{References}

1. O'Connor CJ. Pain relief and pulmonary morbidity after cardiac surgery. Crit Care Med. 1999;27:2314-6.

2. Dowling R, Thielmeier K, Ghaly A, et al. Improved pain control after cardiac surgery: results of a randomized, double-blind, clinical trial. J Thorac Cardiovasc Surg. 2003;126:1271-8.

3. Aybek T, Kessler P, Dogan S, et al. Awake coronary artery bypass grafting: utopia or reality? Ann Thorac Surg. 2003;75:1165-70.

4. Mangano DT, Siliciano D, Hollenberg M, et al. Postoperative myocardial ischemia: therapeutic trials using intensive analgesia following surgery. Anesthesiology. 1992;76:342-53.
5. Anand KJS, Hickey PR. Halothane-morphine compared with highdose sufentanil for anesthesia and postoperative analgesia in neonatal cardiac surgery. N Engl J Med. 1992;326:1-9.

6. Chaney MA. Regional anesthesia for cardiothoracic surgery. Baltimore: Lippincott Williams \& Wilkins; 2002 p. 167.

7. Alhashemi JA, Sharpe MD, Harris CL, et al. Effect of subarachnoid morphine administration on extubation time after coronary artery bypass graft surgery. J Cardiothorac Vasc Anesth. 2000;14:639-44.

8. Chaney MA, Smith KR, Barclay JC, Slogoff S. Large-dose intrathecal morphine for coronary artery bypass grafting. Anesth Analg. 1996;83: 215-22.

9. Royse C, Royse A, Soeding P, et al. Prospective randomized trial of high thoracic epidural analgesia for coronary artery bypass surgery. Ann Thorac Surg. 2003;75:93-100.

10. Priestley MC, Cope L, Halliwell R, et al. Thoracic epidural anesthesia for cardiac surgery: the effects on tracheal intubation time and length of hospital stay. Anesth Analg. 2002;94:275-82.

11. Scott NB, Turfrey DJ, Ray DAA, et al. A prospective randomized study of the potential benefits of thoracic epidural anesthesia and analgesia in patients undergoing coronary artery bypass grafting. Anesth Analg. 2001;93:528-35.

\section{UN THE MOVE?}

Don't miss a single issue of the journal! To ensure prompt service when you change your address, please photocopy and complete the form below.

Please send your change of address notification at least six weeks before your move to ensure continued service. We regret we cannot guarantee replacement of issues missed due to late notification.

\section{JOURNAL TITLE:}

Fill in the title of the journal here.

\section{OLD ADDRESS:}

Affix the address label from a recent issue of the journal here.

\section{NEW ADDRESS:}

Clearly print your new address here.

Name

Address

City/State/ZIP

\section{COPY AND MAIL THIS FORM TO:}

Mosby

Subscription Customer Service

6277 Sea Harbor Dr

Orlando, FL 32887
OR FAX TO:

407-363-9661

N/ Mosby
OR PHONE:

800-654-2452

Outside the U.S., call

407-345-4000 